\title{
Boolean complexes and boolean numbers
}

\author{
Bridget Eileen Tenner \\ Department of Mathematical Sciences, DePaul University, Chicago, Illinois
}

\begin{abstract}
The Bruhat order gives a poset structure to any Coxeter group. The ideal of elements in this poset having boolean principal order ideals forms a simplicial poset. This simplicial poset defines the boolean complex for the group. In a Coxeter system of rank $n$, we show that the boolean complex is homotopy equivalent to a wedge of $(n-1)$ dimensional spheres. The number of these spheres is the boolean number, which can be computed inductively from the unlabeled Coxeter system, thus defining a graph invariant. For certain families of graphs, the boolean numbers have intriguing combinatorial properties. This work involves joint efforts with Claesson, Kitaev, and Ragnarsson.

Résumé. L'ordre de Bruhat munit tout groupe de Coxeter d'une structure de poset. L'idéal composé des éléments de ce poset engendrant des idéaux principaux ordonnés booléens, forme un poset simplicial. Ce poset simplicial définit le complexe booléen pour le groupe. Dans un système de Coxeter de rang $n$, nous montrons que le complexe booléen est homotopiquement équivalent à un bouquet de sphères de dimension $(n-1)$. Le nombre de ces sphères est le nombre booléen, qui peut être calculé inductivement à partir du système de Coxeter non-étiquetté; définissant ainsi un invariant de graphe. Pour certaines familles de graphes, les nombres booléens satisfont des propriétés combinatoires intriguantes. Ce travail est une collaboration entre Claesson, Kitaev, et Ragnarsson.
\end{abstract}

Keywords: Coxeter system, Bruhat order, boolean, boolean number, cell complex, homotopy

\section{Boolean complexes}

The boolean complex of a finitely generated Coxeter system $(W, S)$ was introduced by the author and Ragnarsson in [RT], and the first sections here describe this work. The boolean Complex arises from the (strong) Bruhat order on $W$. This ordering gives a poset structure to $W$, and the boolean ideal $\mathbb{B}(W, S) \subseteq W$ is the subposet comprised of those elements of $W$ whose principal order ideals are boolean. Such elements are boolean elements. This order ideal $\mathbb{B}(W, S)$ is necessarily a simplicial poset.

Definition 1.1 Let $(W, S)$ be a Coxeter system. The boolean complex of $(W, S)$ is the regular cell complex $\Delta(W, S)$ whose face poset is the simplicial poset $\mathbb{B}(W, S)$.

The existence of the complex $\Delta(W, S)$ follows from a result of Björner about simplicial posets, and in fact about CW-posets (see [Bjö]).

The following lemma is immediate from the definition of the Bruhat order, and gives a useful characterization of boolean elements.

Lemma 1.2 Let $(W, S)$ be a Coxeter system. An element of $W$ is boolean if and only if it has no repeated letters in its reduced expressions.

1365-8050 @ 2010 Discrete Mathematics and Theoretical Computer Science (DMTCS), Nancy, France 
It follows from Lemma 1.2 that the boolean complex is pure, and each maximal face has dimension $|S|-1$.

The relevance of boolean elements arises from their properties related to $R$-polynomials, KazhdanLusztig polynomials, and $g$-polynomials (see [Bre]).

The fact that the boolean elements are exactly those which are the products of distinct elements of the generating set $S$ means that these elements are described by the commutativity of elements of $S$. This commutativity information is encoded in the unlabeled Coxeter graph of $(W, S)$, and thus the boolean objects described above can be reformulated in terms of graphs. This graph-theoretic description is given in the following paragraphs, and also appears in work of Jonsson and Welker [JW].

Let $G$ be a finite simple graph, and let $S$ be the vertex set of $G$. Set $\mathbb{W}(S)$ to be the set of words on $S$ with no repeated letters, ordered by the subword order relation. Elements of $\mathbb{W}(S)$ are thus of the form $s_{1} s_{2} \cdots s_{\ell}$, where $s_{1}, s_{2}, \ldots, s_{\ell} \in S$ are distinct elements. We generate an equivalence relation by the requirement that

$$
s_{1} \cdots s_{i-1} s_{i} s_{i+1} s_{i+2} \cdots s_{\ell} \sim s_{1} \cdots s_{i-1} s_{i+1} s_{i} s_{i+2} \cdots s_{\ell}
$$

if $\left\{s_{i}, s_{i+1}\right\}$ is not an edge in $G$.

The equivalence relation generated by this $\sim$ defines a set of equivalence classes on $\mathbb{W}(S)$, which we call $\mathbb{B}(G)$. The preimages of an element $\sigma \in \mathbb{B}(G)$ are word representatives. Note that the set of letters occurring in each word representative of $\sigma$ is the same. A poset structure on $\mathbb{B}(G)$ arises from the subword order: $\sigma \leq \tau$ in $\mathbb{B}(G)$ if some word representative of $\sigma$ is a subword of a word representative of $\tau$.

A special case worthy of its own mention is the complete graph $K_{n}$. For this graph, the ideal $\mathbb{B}\left(K_{n}\right)$ describes the complex of injective words. This complex has been studied by Farmer [Far], Björner and Wachs [BW], and Reiner and Webb [RW]. The complete graph is treated here in Corollary 4.5

Example 1.3 For the complete graph $K_{2}$, the poset $\mathbb{B}\left(K_{2}\right)$ and the boolean complex $\Delta\left(K_{2}\right)$ are depicted in Figure 1

(a)

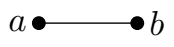

(b)

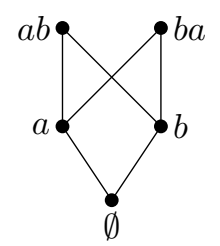

(c)

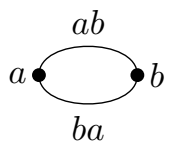

Fig. 1: (a) The graph $K_{2}$. (b) The poset $\mathbb{B}\left(K_{2}\right)$. (c) The boolean complex $\Delta\left(K_{2}\right)$, whose geometric realization $\left|\Delta\left(K_{2}\right)\right|$ is homotopy equivalent to $S^{1}$.

The unlabeled Coxeter graphs of the Coxeter groups $A_{2}, B_{2} / C_{2}, G_{2}$ and $I_{2}(m)$ are all the same as $K_{2}$. Thus Example 1.3 demonstrates that the geometric realization of each of these boolean complexes is homotopy equivalent to $S^{1}$. More generally, as shown in joint work with Ragnarsson in [RT], and discussed in Section 3, the boolean complex of any finite simple graph, and hence of any Coxeter system, has a similar property: its geometric realization is homotopy equivalent to a wedge of top dimensional spheres, and we give a recursive formula for calculating the number of these spheres. 


\section{Motivation and definitions}

This analysis of boolean complexes and their topological and enumerative properties is motivated by the field of Coxeter systems and the relevance of their boolean elements.

Throughout this work, we assume that all Coxeter systems are finitely generated.

The Bruhat order makes a Coxeter group $W$ into a ranked poset, with rank determined by the number of letters in a reduced expression of an element. We use the convention that the minimal element in the poset $\mathbb{B}(W, S)$ has rank -1 , because this element corresponds to the empty face in the geometric realization of that poset. This reinforces the fact that the face data of the complex is described by the non-negative ranks in the poset. More precisely, an element of rank $k \geq 0$ represents a $k$-dimensional cell.

Although one can view the cells in $\mathbb{B}(W, S)$ as simplices, because the minimal subcomplex containing each cell is isomorphic to a simplex of the same dimension, it must be noted that the boolean complex itself is not a simplicial complex. This is because the cells in the complex are not uniquely determined by the vertices they contain. One case of this is depicted in Example 1.3, where two 1-cells have the same vertices.

A geometric realization $|\Delta(W, S)|$ of the boolean complex $\Delta(W, S)$ is obtained in the standard way: we take one geometric simplex of dimension $k$ for each cell of dimension $k$, and glue them together according to the data encoded in the face poset. The homotopy type of this geometric realization is what we mean when we say the homotopy type of a boolean complex.

Theorem 3.4 states the main result in this area, which is that $|\Delta(W, S)|$ has the homotopy type of a wedge of spheres of dimension $|S|-1$. Moreover, the number of spheres in the wedge can be computed by a recursive formula, also given in the theorem. This recursion is given in terms of graph operations, and uses the alternative construction of the boolean complex, in terms of the unlabeled Coxeter graph of $(W, S)$. The motivation for the construction of $\mathbb{B}(G)$ is the following obvious fact: if $G$ is the unlabeled Coxeter graph of the Coxeter system $(W, S)$, then $\mathbb{B}(G) \cong \mathbb{B}(W, S)$.

Definition 2.1 The boolean complex of a finite simple graph $G$ is the regular cell complex $\Delta(G)$ associated to $\mathbb{B}(G)$.

The main result about boolean complexes for Coxeter systems can be restated as follows: for any finite simple graph $G$ with vertex set $S$, the geometric realization $|\Delta(G)|$ is homotopy equivalent to a wedge of $(|S|-1)$-dimensional spheres. It is this version of the result which we shall state, and, as promised, the recursive formula for the number of spheres is given in terms of basic graph operations on $G$.

Before discussing the main results, we foreshadow one of them for the Coxeter group $A_{n}$. The Euler characteristic $\chi$ of a regular cell complex $\Delta$, and likewise the Euler characteristic of its geometric realization $|\Delta|$, is the alternating sum of the number of faces of each non-negative rank in $\Delta$. Given that we consider the rank of the minimal element in a Coxeter group to be -1 , this can be computed by an alternating sum of the number of elements in each non-negative rank in the corresponding simplicial poset. In particular, an enumeration from [Ten] enables the calculation of the Euler characteristic of the boolean complex for the Coxeter group $A_{n}$.

Corollary 2.2 For all $n \geq 1$,

$$
\chi\left(\Delta\left(A_{n}\right)\right)=(-1)^{n-1} f(n-1)+1,
$$

where $\{f(n)\}$ are the Fibonacci numbers. 
It is convenient to use the notation $b \cdot S^{r}$ for a wedge sum of $b$ spheres of dimension $r$. Since the wedge sum is the coproduct in the category of pointed spaces, $0 \cdot S^{r}$ then denotes a single point. Corollary 2.2 foreshadows the fact that $\left|\Delta\left(A_{n}\right)\right|$ is homotopy equivalent to the wedge sum $f(n-1) \cdot S^{n-1}$. Although the statistic discussed in this work is equal to the absolute value of the reduced Euler characteristic, Corollary 2.2 references the unreduced Euler characteristic because it is valuable to point out the relationship between $\left\{\chi\left(\Delta\left(A_{n}\right)\right)\right\}$ and sequences $\mathrm{A} 008346$ and $\mathrm{A} 119282$ in [Slo]: sequence A008346 is equal to $\left\{\left|\chi\left(\Delta\left(A_{n}\right)\right)\right|\right\}$, while sequence $\mathrm{A} 119282$ is equal to $\left\{-\chi\left(\Delta\left(A_{n}\right)\right)\right\}$.

\section{Computing the homotopy type}

We now state the main result about the homotopy type of the boolean complex. As mentioned previously, this is stated in terms of the graph formulation (thus it is a statement about $\Delta(G)$ ) because of a recursive formula involving graph operations.

Definition 3.1 For a finite graph $G$, let $|G|$ denote the number of vertices in $G$.

Definition 3.2 Let $G$ be a finite simple graph and e an edge in $G$. Define three operations on $G$ as follows.

- Deletion: $G-e$ is the graph obtained by deleting the edge e.

- Simple contraction: G/e is the graph obtained by contracting the edge e and then removing all loops and redundant edges.

- Extraction: $G-[e]$ is the graph obtained by removing the edge $e$ and its incident vertices.

Definition 3.3 For $n \geq 1$, let $\delta_{n}$ be the graph consisting of $n$ disconnected vertices.

The symbol $\simeq$ will denote homotopy equivalence in the following theorem and subsequent discussion.

Theorem 3.4 ([|RT]) For every nonempty, finite simple graph $G$, there is an integer $\beta(G)$ so that

$$
|\Delta(G)| \simeq \beta(G) \cdot S^{|G|-1} .
$$

Moreover, the values $\beta(G)$ can be computed using the recursive formula

$$
\beta(G)=\beta(G-e)+\beta(G / e)+\beta(G-[e]),
$$

if $e$ is an edge in $G$ such that $G-[e]$ is nonempty, with initial conditions

$$
\beta\left(K_{2}\right)=1 \text { and } \beta\left(\delta_{n}\right)=0 .
$$

The integer $\beta(G)$ is the boolean number of the graph $G$.

Because $\mathbb{B}(G) \cong \mathbb{B}(W, S)$ when $G$ is the unlabeled Coxeter graph of the Coxeter system $(W, S)$, Theorem 3.4 implies that the geometric realization of the boolean complex of a Coxeter system $(W, S)$ is homotopy equivalent to a wedge of spheres of dimension $|S|-1$. The number of spheres occurring in the wedge can be calculated recursively using equation (1). In fact, this calculation can be aided by the next result, which states that the boolean number is multiplicative with respect to connected components of a graph. 
Proposition 3.5 ([|RT]) If $G=H_{1} \sqcup H_{2}$ for graphs $H_{1}$ and $H_{2}$, then

$$
\Delta(G)=\Delta\left(H_{1}\right) * \Delta\left(H_{2}\right),
$$

where $*$ denotes simplicial join. Thus

$$
|\Delta(G)| \simeq \beta\left(H_{1}\right) \beta\left(H_{2}\right) \cdot S^{\left|H_{1}\right|+\left|H_{2}\right|-1} .
$$

In particular, $\beta(G)=\beta\left(H_{1}\right) \beta\left(H_{2}\right)$.

\section{Homotopy type in special cases}

For some classes of graphs, we can obtain more specific data regarding the boolean number $\beta$. A selection of these results are given below.

If the graph $G$ has any leaves, then the recursive equation (1) can be simplified by Proposition 3.5

Corollary 4.1 If $G$ has a vertex of degree one, then the computation of $\beta(G)$ is simplified according to the identities in Figure 2

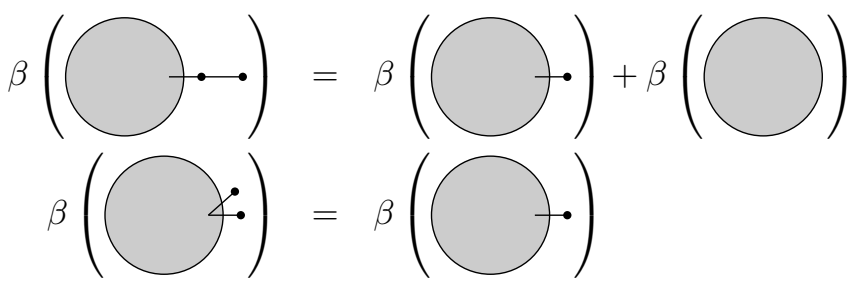

Fig. 2: Simplification for recursively calculating $\beta(G)$ when the graph $G$ has a leaf.

Combining the results of Corollary 4.1 and Theorem 3.4 allows for the efficient calculation of the boolean number of trees. Moreover, it shows that if $T$ is a tree with more than one vertex, then $\beta(T)>0$. The recursive formula for calculating $\beta$ shows that adding edges does not decrease the boolean number, which leads to the following conclusion.

Corollary $4.2([\mathbf{R T} \mid)$ A finite simple graph $G$ has an isolated vertex if and only if $\beta(G)=0$.

In other words, the boolean complex of a Coxeter group is contractible if and only if the center of the group contains a generator of the group.

Definition 4.3 For $n \geq 1$, let $S_{n}$ be the star graph on $n$ vertices.

Corollary 4.4 For $n \geq 1, \beta\left(S_{n}\right)=1$. That is, $\Delta\left(S_{n}\right) \simeq S^{n-1}$.

Recursively computing the boolean numbers of complete graphs is similarly easy, and gives a recurrence indicating the following corollary.

Corollary 4.5 For $n \geq 1, \beta\left(K_{n}\right)$ is equal to the number of derangements of $[n]$. 
Corollary 4.5 recovers a result of Reiner and Webb, which was proved in the context of the complex of injective words, using character theory $[\mathrm{RW}]$.

Equation (11) indicates that the function $\beta$ is monotonically increasing with respect to edge addition. More precisely, if $H \subseteq G$ is obtained by deleting some edges from the graph $G$, then $\beta(H) \leq \beta(G)$. One could ask when this inequality is strict, and when there is equality.

Corollary 4.6 ([|RT|) Fix a finite simple graph $G$. Obtain $H \subset G$ by removing an edge of $G$. Then $\beta(H)=\beta(G)$ if and only if $G$ has an isolated vertex, in which case $\beta(G)=\beta(H)=0$. Otherwise $\beta(H)<\beta(G)$.

As suggested earlier, the boolean number for the Coxeter group $A_{n}$ is the Fibonacci number $f(n-1)$. Given Corollaries 4.1, 4.4, and 4.6, we see that among all trees on $n$ vertices, the smallest boolean number is obtained by the star $S_{n}$, and the largest boolean number is obtained by the path $A_{n}$.

\section{Boolean numbers: enumerative directions}

Given a finite simple graph $G$, the boolean number $\beta(G)$ is a graph invariant. There are a variety of enumerative aspects of this object that one can explore, several of which are mentioned here.

Corollary 4.6 gives some perspective to the image of the function $\beta$. If we analyze the function $\beta$, we quickly find that it is neither surjective nor injective, in the following senses. Let $\mathcal{G}_{n}$ be the set of finite simple graphs having $n$ vertices. The set $\left\{\beta(G): G \in \mathcal{G}_{n}\right\}$ is not necessarily a contiguous interval of values (necessarily beginning at 0 ), and it may be that $\beta(G)=\beta(H)$ for distinct graphs $G, H \in \mathcal{G}_{n}$.

Example 5.1 No graph in $\mathcal{G}_{4}$ has boolean number 4, although the boolean number of a 4-cycle is 5 .

Example 5.2 The two graphs in Figure 3 each have boolean number 3, and thus are each homotopy equivalent to $S^{4} \vee S^{4} \vee S^{4}$.
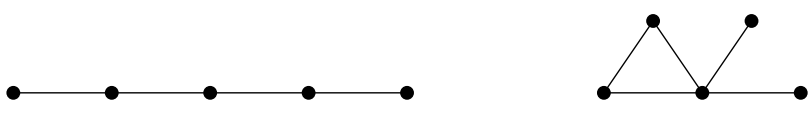

Fig. 3: Two elements of $\mathcal{G}_{5}$, each having boolean number 3 .

As suggested already, there are several families of graphs whose boolean numbers give well-known sequences. Three such families already mentioned are

- graphs with disjoint vertices (boolean numbers are always 0 ),

- paths (boolean numbers are the Fibonacci numbers), and

- complete graphs (boolean numbers are the derangement numbers).

Other interesting connections to integer sequences have arisen in joint work with Claesson, Kitaev, and Ragnarsson [CKRT]. These results are related to Ferrers graphs, particularly for rectangular and staircase shapes, and are described below.

As described in [CN], Ferrers shapes, or Young shapes or partitions, arise in a variety of contexts including Schubert varieties, symmetric functions, hypergeometric series, permutation statistics, quantum 
mechanical operators, and inverse rook problems. One can relate a bipartite graph known as a Ferrers graph to any Ferrers shape, as introduced in [EvW].

Definition 5.3 Let $\lambda=\left(\lambda_{1}, \ldots, \lambda_{r}\right)$ be a partition, where $\lambda_{1} \geq \cdots \geq \lambda_{r} \geq 0$. The associated bipartite Ferrers graph has vertices $\left\{x_{1}, \ldots, x_{r}\right\} \sqcup\left\{y_{1}, \ldots, y_{\lambda_{1}}\right\}$, and edges $\left\{\left\{x_{i}, y_{j}\right\}: \lambda_{i} \geq j\right\}$.

In particular, vertex $x_{i}$ has degree $\lambda_{i}$. A Ferrers graph and its associated Ferrers shape are depicted in Figure 4
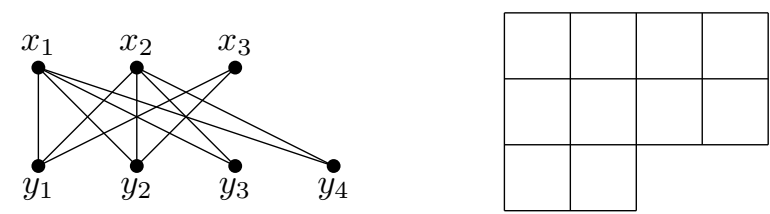

Fig. 4: The Ferrers graph and shape for the partition $\lambda=(4,4,2)$.

The Ferrers graph of an $m$-by- $n$ rectangular shape is the complete bipartite graph $K_{m, n}$. Computation of the boolean number of such a graph invokes the Stirling numbers of the second kind.

Corollary 5.4 ([CKRT] $)$ For $m, n \geq 1$,

$$
\beta\left(K_{m, n}\right)=\sum_{k=1}^{m}(-1)^{m-k} k !\left\{\begin{array}{c}
m+1 \\
k+1
\end{array}\right\} k^{n} .
$$

Even more directly, the boolean numbers of the Ferrers graph of staircase shapes are exactly the median Genocchi numbers, which are sequence A005439 of [Slo]. As shown in [ES], the median Genocchi number $g_{n}$ is equal to the number of permutations of $2 n$ letters having alternating excedances.

Definition 5.5 For $n \geq 1$, the staircase shape of height $n$ is the Ferrers shape

$$
\Sigma_{n}=(n, n-1, \ldots, 2,1) .
$$

Let $F_{n}$ denote the Ferrers graph for the Ferrers shape $\Sigma_{n}$.

Corollary 5.6 ([CKRT]) For $n \geq 1, \beta\left(F_{n}\right)=g_{n}$.

\section{References}

[Bjö] A. Björner, Posets, regular CW complexes and Bruhat order, European J. Combin. 5 (1984), 7-16.

[BW] A. Björner and M. Wachs, On lexicographically shellable posets, Trans. Amer. Math. Soc. 277 (1983), 323-341.

[Bre] F. Brenti, A combinatorial formula for Kazhdan-Lusztig polynomials, Invent. Math. 118 (1994), 371-394. 
[CKRT] A. Claesson, S. Kitaev, K. Ragnarsson, and B. E. Tenner, Boolean complexes for Ferrers graphs, preprint.

[CN] A. Corso and U. Nagel, Monomial and toric ideals associated to Ferrers graphs, Trans. Amer. Math. Soc., 361 (2009), 1371-1395.

[ES] R. Ehrenborg and E. Steingrímsson, The excedance set of a permutation, Adv. Appl. Math. 24 (2000), 284-299.

[EvW] R. Ehrenborg and S. van Willigenburg, Enumerative properties of Ferrers graphs, Discrete Comput. Geom. special issue in honor of Louis J. Billera, 32 (2004), 481-492.

[Far] F. D. Farmer, Cellular homology for posets, Math. Japon. 23 (1978/79), 607-613.

[JW] J. Jonsson and V. Welker, Complexes of injective words and their commutation classes, Pacific J. Math. 243 (2009), 313-329.

[RT] K. Ragnarsson and B. E. Tenner, Homotopy type of the boolean complex of a Coxeter system, Adv. Math. 222 (2009), 409-430.

[RW] V. Reiner and P. Webb, The combinatorics of the bar resolution in group cohomology, J. Pure Appl. Algebra 190 (2004), 291-327.

[Slo] N. J. A. Sloane, The on-line encyclopedia of integer sequences, published electronically at http:/www.research.att.com/njas/sequences/.

[Sta] R. P. Stanley, Enumerative Combinatorics, vol. 1, Cambridge Studies in Advanced Mathematics, no. 49, Cambridge University Press, Cambridge, 1997

[Ten] B. E. Tenner, Pattern avoidance and the Bruhat order, J. Combin. Theory, Ser. A 114 (2007), 888-905. 